\title{
diffusion-fundarnentals.org
}

The Open-Access Journal for the Basic Principles of Diffusion Theory, Experiment and Application

\section{Insights from a Nanoparticle Minuet: Two-Dimensional Membrane Profiling through Silver Plasmon Ruler Tracking}

\author{
Guoxin Rong, Hongyun Wang, and Björn M. Reinhard*
}

Department of Chemistry and The Photonics Center, Boston University, Boston, Massachusetts 02215, United States E-Mail: gxrong@bu.edu

\section{Introduction}

"The plasma membrane of mammalian cells represents a complex two dimensional diffusion system, where the spatial organization and dynamics of both lipids and proteins plays an important role in regulating signal transduction and membrane traffic. [1] Important insights into the organization of cellular surfaces are available through particle tracking experiments.

Conventional high-speed particle tracking is however limited because of the diffraction limit of $\sim 400 \mathrm{~nm}$ in the visible. We have recently shown that this limitation can be overcome by utilizing the distance dependent plasmon coupling between individual noble metal nanoparticles. [2] Here we

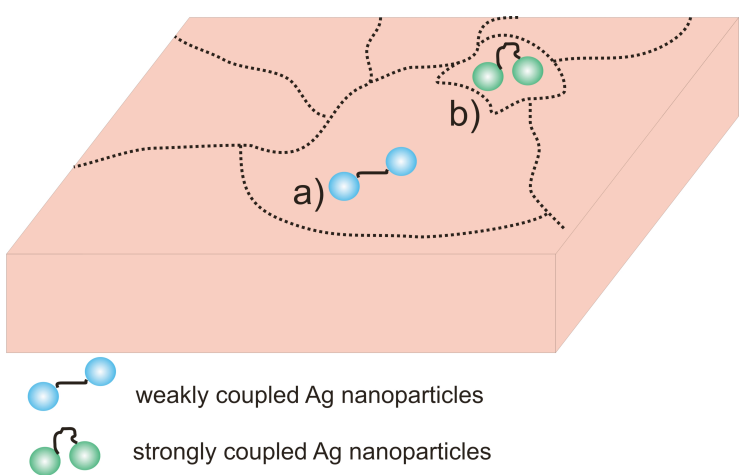

Figure 1: Pairs of individual polymer tethered nanoparticles serve as probes in polarization resolved plasmon coupling microscopy (PRPCM) to probe the spatial organization of the plasma membrane. For dimers that are trapped in attractive sites on the membrane surface, the wavelength and the polarization of the scattered light provide additional information about the interparticle separation and the rotational freedom of the dimers in these sites. The average interparticle separation and mobility of the plasmon rulers is expected to decrease with increasing confinement as illustrated for examples a) and b).

take advantage of the

distance and orientation dependent optical properties of pre-assembled silver dimers, so called silver plasmon rulers as probes in particle tracking. [3] Our aim is to apply these active nanostructures to map the two-dimensional organization of mammalian plasma membranes on sub-micron length scales. (Fig. 1) 


\section{Polarization Resolved and Ratiometric Tracking of Silver Plasmon Rulers}

The reduced linear dichroism and the spectrum of the light scattered from individual plasmon rulers encode information about the structural details of a plasmon ruler's confinement region. We analyzed the translational and rotational motions as well as the extension of individual silver plasmon rulers diffusing on the plasma membrane of lysed HeLa cells. This approach, which we refer to as polarization-resolved plasmon coupling microscopy (PRPCM), provided a quantitative metrics to characterize the structural lateral heterogeneity of cell membranes on submicrometer length scales as a function of time. In combination with adequate tracking methods, the "dance" performed by membrane confined dimers of flexibly linked noble metal nanoparticles revealed deep insight into the underlying membrane organization. [3]

\section{Conclusion}

Using a polarization resolved tracking approach we monitored the translational and rotational mobility of individual silver plasmon rulers on lysed $\mathrm{HeLa}$ cells with a temporal resolution of $500 \mathrm{~Hz}$. The analysis of the translational motion of the individual plasmon rulers revealed that most of the plasmon rulers are either immobilized or undergo a confined diffusion on the cell surface. Those plasmon rulers that undergo confined diffusion show large differences in the rotational dynamics which was attributed to the lateral heterogeneity of the membrane. To obtain additional information about the compartmentalization of the membrane, we implemented the PRPCM that enabled us to track individual silver plasmon rulers diffusing on the surface of lysed HeLa cells and simultaneously monitor the plasmon rulers' orientation and interparticle separation. Consistent with a compartmentalization of the membrane on length scales of the plasmon rulers (dimers of $30 \mathrm{~nm}$ particles with center to center separation of $\sim 44 \mathrm{~nm}$ ) we find that confinement of the plasmon rulers on the cell surface affects their rotational freedom and average interparticle separation. We demonstrated that by mapping interparticle separation and polarization on the cell surface, detailed insight into the cell membrane organization with nanometer scale spatial precison becomes accessible. The gain in information in polarization resolved ratiometric tracking when compared with conventional particle tracking makes PRPCM a promising tool for analyzing the lateral heterogeneity of complex cellular surfaces." [3]

\section{References}

[1] A. Whitty, Nature Chem Bio. 4(2008) 435-439

[2] G. Rong, H. Wang, L.R. Skewis, B. M. Reinhard, Nano Lett. 8(2008) 3386 - 3393

[3] G. Rong, H. Wang, B. M. Reinhard, Nano. Lett. 10 (2010) 230-238. 